\title{
RICCI-LIKE SOLITONS WITH VERTICAL POTENTIAL ON SASAKI-LIKE ALMOST CONTACT B-METRIC MANIFOLDS
}

\author{
MANCHO MANEV
}

\begin{abstract}
Ricci-like solitons on Sasaki-like almost contact B-metric manifolds are the object of study. Cases, where the potential of the Ricci-like soliton is the Reeb vector field or pointwise collinear to it, are considered. In the former case, the properties for a parallel or recurrent Ricci-tensor are studied. In the latter case, it is shown that the potential of the considered Ricci-like soliton has a constant length and the manifold is $\eta$-Einstein. Other curvature conditions are also found, which imply that the main metric is Einstein. After that, some results are obtained for a parallel symmetric second-order covariant tensor on the manifolds under study. Finally, an explicit example of dimension 5 is given and some of the results are illustrated.
\end{abstract}

\section{INTRODUCTION}

Following the R.S. Hamilton's concept of a Ricci flow, introduced in 1982 [11], R. Sharma initiated the study of Ricci solitons in contact Riemannian geometry, particularly on K-contact manifolds in 2008 [23]. After that Ricci solitons have been studied on different kinds of almost contact metric manifolds, e.g. $\alpha$-Sasakian [12], trans-Sasakian [2], Kenmotsu [20], etc.

Although this topic was first studied in Riemannian geometry, in recent years Ricci solitons and their generalizations have also been studied in pseudo-Riemannian manifolds, mostly with Lorentzian metrics ([1], [3], [4], [19], [24]).

Almost contact metric manifolds are usually equipped with a compatible metric, which can be Riemannian or pseudo-Riemannian. Then, the contact $(1,1)$-tensor $\varphi$ acts as an isometry with respect to the metric on the contact distribution. The alternative case is when this action is an anti-isometry. Then, the metric is necessarily pseudo-Riemannian and it is known as a B-metric. Moreover, the associated $(0,2)$-tensor of a B-metric with respect to $\varphi$ is also a B-metric, in contrast to the associated $(0,2)$-tensor of a compatible metric is a 2 -form. The differential geometry of almost contact B-metric manifolds has been studied since 1993 [8], [18].

In our previous work [16], we introduced the notion of Ricci-like solitons on almost contact B-metric manifolds as a generalization of Ricci solitons and $\eta$-Ricci solitons using both B-metrics of manifold. There, we investigated the case of a potential Reeb vector field, when either the manifold is Sasaki-like or the Reeb vector field is torse-forming.

2010 Mathematics Subject Classification. Primary 53C25, 53D15, 53C50; Secondary 53C44, 53D35, $70 \mathrm{G} 45$.

Key words and phrases. Ricci-like soliton, $\eta$-Ricci soliton, Einstein-like manifold, $\eta$-Einstein manifold, almost contact B-metric manifold, almost contact complex Riemannian manifold. 
In the present paper, our goal is to continue our study on Ricci-like solitons, a generalization of Ricci solitons compatible with the almost contact B-metric structure. Now we focus on the case, when the potential of the considered Ricci-like soliton is a vertical vector field, i.e. it is orthogonal to the contact distribution with respect to the metric. The paper is organized as follows. Section 1 is devoted to the basic concepts of almost contact B-metric manifolds of Sasaki-like type. In Section 2, we study the covariant derivative of the Ricci tensor with respect to the main B-metric $g$ of the manifold with a Ricci-like soliton, which potential is exactly the Reeb vector field $\xi$. In Section 3, we consider Ricci-like solitons with potential, which is the Reeb vector field multiplied by a differentiable function $k$. Then, we prove that $k$ is constant and the Sasaki-like manifold is $\eta$-Einstein. Also a series of curvature conditions of the manifold are considered that imply $k$ is a constant and $g$ is an Einstein metric. In Section 4, we give some characterization for Ricci-like solitons on Sasaki-like manifold concerning a parallel symmetric $(0,2)$-tensor. In Section 5, we give an explicit example of a Lie group of dimension 5 equipped with a Sasaki-like almost contact B-metric structure. Then, we show that the manifold is $\eta$-Einstein of a special type, admits a Ricci-like soliton with potential $\xi$ and the results for this example support the relevant assertions in the previous sections.

\section{SASAKI-LiKe Almost CONTACT B-MetriC MANiFOLdS}

We consider almost contact B-metric manifolds. A differentiable manifold $M$ of this type has dimension $(2 n+1)$ and it is denoted by $(M, \varphi, \xi, \eta, g)$, where $(\varphi, \xi, \eta)$ is an almost contact structure and $g$ is a B-metric. More precisely, $\varphi$ is an endomorphism of the tangent bundle $T M, \xi$ is a Reeb vector field, $\eta$ is its dual contact 1 -form and $g$ is a pseudo-Riemannian metric $g$ of signature $(n+1, n)$ satisfying the following conditions [8]

$$
\begin{aligned}
\varphi \xi=0, \quad & \varphi^{2}=-\iota+\eta \otimes \xi, \quad \eta \circ \varphi=0, \quad \eta(\xi)=1, \\
& g(\varphi x, \varphi y)=-g(x, y)+\eta(x) \eta(y),
\end{aligned}
$$

where $\iota$ is the identity transformation on $\Gamma(T M)$.

In the latter equality and further, $x, y, z, w$ will stand for arbitrary elements of $\Gamma(T M)$ or vectors in the tangent space $T_{p} M$ of $M$ at an arbitrary point $p$ in $M$.

Some immediate consequences of (1.1) are the following equations

$$
\begin{array}{ll}
g(\varphi x, y)=g(x, \varphi y), & g(x, \xi)=\eta(x), \\
g(\xi, \xi)=1, & \eta\left(\nabla_{x} \xi\right)=0,
\end{array}
$$

where $\nabla$ is the Levi-Civita connection of $g$.

The associated metric $\tilde{g}$ of $g$ on $M$ is defined by

$$
\tilde{g}(x, y)=g(x, \varphi y)+\eta(x) \eta(y) .
$$

It is also a B-metric.

A classification of almost contact B-metric manifolds, consisting of eleven basic classes $\mathcal{F}_{i}, i \in\{1,2, \ldots, 11\}$, is given in [8]. This classification is made with respect to the $(0,3)$-tensor $F$ defined by

$$
F(x, y, z)=g\left(\left(\nabla_{x} \varphi\right) y, z\right) .
$$


It possess the following basic properties:

$$
\begin{aligned}
& F(x, y, z)=F(x, z, y)=F(x, \varphi y, \varphi z)+\eta(y) F(x, \xi, z)+\eta(z) F(x, y, \xi), \\
& F(x, \varphi y, \xi)=\left(\nabla_{x} \eta\right) y=g\left(\nabla_{x} \xi, y\right) .
\end{aligned}
$$

The intersection of the basic classes is the special class $\mathcal{F}_{0}$, determined by the condition $F=0$, and it is known as the class of the cosymplectic B-metric manifolds.

The Lee forms of $(M, \varphi, \xi, \eta, g)$ are the following 1-forms associated with $F$ :

$$
\theta(z)=g^{i j} F\left(e_{i}, e_{j}, z\right), \quad \theta^{*}(z)=g^{i j} F\left(e_{i}, \varphi e_{j}, z\right), \quad \omega(z)=F(\xi, \xi, z),
$$

where $\left(g^{i j}\right)$ is the inverse matrix of the matrix $\left(g_{i j}\right)$ of $g$ with respect to a basis $\left\{e_{i} ; \xi\right\}(i=1,2, \ldots, 2 n)$ of $T_{p} M$. Obviously, $\omega(\xi)=\theta^{*} \circ \varphi+\theta \circ \varphi^{2}=0$ are valid.

In [13], it is introduced the type of Sasaki-like manifolds among almost contact B-metric manifolds (also known as almost contact complex Riemannian manifolds). The definition condition is its complex cone to be a Kähler-Norden manifold, i.e. with a parallel complex structure. A Sasaki-like manifold with almost contact Bmetric structure is determined by the condition

$$
\left(\nabla_{x} \varphi\right) y=-g(x, y) \xi-\eta(y) x+2 \eta(x) \eta(y) \xi
$$

or equivalently $\left(\nabla_{x} \varphi\right) y=g(\varphi x, \varphi y) \xi+\eta(y) \varphi^{2} x$.

Obviously, any Sasaki-like manifold belongs to the class $\mathcal{F}_{4}$ and its Lee forms are $\theta=-2 n \eta, \theta^{*}=\omega=0$. Moreover, the following identities are valid for it [13]

$$
\begin{array}{ll}
\nabla_{x} \xi=-\varphi x, & \left(\nabla_{x} \eta\right)(y)=-g(x, \varphi y), \\
R(x, y) \xi=\eta(y) x-\eta(x) y, & \rho(x, \xi)=2 n \eta(x), \\
R(\xi, y) z=g(y, z) \xi-\eta(z) y, & \rho(\xi, \xi)=2 n,
\end{array}
$$

where $R$ and $\rho$ stand for the curvature tensor and the Ricci tensor of $\nabla$.

Let $\tau$ and $\tilde{\tau}$ be the scalar curvatures with respect to $g$ and $\tilde{g}$, respectively, and let $\tau^{*}$ be the associated quantity of $\tau \operatorname{regarding} \varphi$, defined by $\tau^{*}=g^{i j} \rho\left(e_{i}, \varphi e_{j}\right)$.

For an arbitrary $\mathcal{F}_{4}$-manifold with a closed 1 -form $\theta$, the following relation for $\tau^{*}$ and $\tilde{\tau}$ is given in [15]

$$
\tilde{\tau}=-\tau^{*}+\frac{1}{2 n}(\theta(\xi))^{2}+\xi(\theta(\xi)) .
$$

Then, for a Sasaki-like manifold since $\theta(\xi)=-2 n$ we have

$$
\tilde{\tau}=-\tau^{*}+2 n
$$

Let us recall [16], an almost contact $\mathrm{B}$-metric manifold $(M, \varphi, \xi, \eta, g)$ is said to be Einstein-like if its Ricci tensor $\rho$ satisfies

$$
\rho=a g+b \tilde{g}+c \eta \otimes \eta
$$

for some triplet of constants $(a, b, c)$ [16]. In particular, when $b=0$ and $b=$ $c=0$, the manifold is called an $\eta$-Einstein manifold and an Einstein manifold, respectively.

Tracing (1.8), the scalar curvature $\tau$ of an Einstein-like almost contact B-metric manifold has the form

$$
\tau=(2 n+1) a+b+c
$$


For a Sasaki-like manifold $(M, \varphi, \xi, \eta, g)$ with $\operatorname{dim} M=2 n+1$ and a scalar curvature $\tau$ regarding $g$, which is Einstein-like with a triplet of constants $(a, b, c)$, the following equalities are given in [16]:

$$
a+b+c=2 n, \quad \tau=2 n(a+1) .
$$

Then, for $\tilde{\tau}$ on an Einstein-like Sasaki-like manifold we obtain

$$
\tilde{\tau}=2 n(b+1)
$$

and because (1.9), (1.10) and (1.11), the expression (1.8) becomes

$$
\rho=\left(\frac{\tau}{2 n}-1\right) g+\left(\frac{\tilde{\tau}}{2 n}-1\right) \tilde{g}+\left(2(n+1)-\frac{\tau+\tilde{\tau}}{2 n}\right) \eta \otimes \eta .
$$

Proposition $1.1([17])$. Let $(M, \varphi, \xi, \eta, g)$ be a $(2 n+1)$-dimensional Sasaki-like manifold. If it is almost Einstein-like with functions $(a, b, c)$ then the scalar curvatures $\tau$ and $\tilde{\tau}$ of $g$ and $\tilde{g}$, respectively, are constants

$$
\tau=\text { const }, \quad \tilde{\tau}=2 n
$$

and $(M, \varphi, \xi, \eta, g)$ is $\eta$-Einstein with constants

$$
(a, b, c)=\left(\frac{\tau}{2 n}-1,0,2 n+1-\frac{\tau}{2 n}\right) .
$$

\section{RicCi-Like solitons With Potential REeB VeCtor Field on SASAKI-LIKE MANIFOLDS}

In [16], by conditions for Ricci tensor, there are introduced the notions of an Einstein-like almost contact B-metric manifold and a Ricci-like soliton on an almost contact B-metric manifold.

It is said that $(M, \varphi, \xi, \eta, g)$ admits a Ricci-like soliton with potential $\xi$ if the following condition is satisfied for a triplet of constants $(\lambda, \mu, \nu)$

$$
\frac{1}{2} \mathcal{L}_{\xi} g+\rho+\lambda g+\mu \tilde{g}+\nu \eta \otimes \eta=0,
$$

where $\mathcal{L}$ denotes the Lie derivative.

If $\mu=0$ (respectively, $\mu=\nu=0$ ), then (2.1) defines an $\eta$-Ricci soliton (respectively, a Ricci soliton) on $(M, \varphi, \xi, \eta, g)$.

If $\lambda, \mu, \nu$ are functions on $M$, then the soliton is called almost Ricci-like soliton, almost $\eta$-Ricci soliton and almost Ricci soliton, respectively.

Theorem 2.1 ([16]). Let $(M, \varphi, \xi, \eta, g)$ be a $(2 n+1)$-dimensional Sasaki-like manifold and let $a, b, c, \lambda, \mu, \nu$ be constants that satisfy the following equalities:

$$
a+\lambda=0, \quad b+\mu-1=0, \quad c+\nu+1=0 .
$$

Then, the manifold admits a Ricci-like soliton with potential $\xi$ and constants $(\lambda, \mu$, $\nu)$, where $\lambda+\mu+\nu=-2 n$, if and only if it is Einstein-like with constants $(a, b, c)$, where $a+b+c=2 n$.

In particular, we get:

(i) The manifold admits an $\eta$-Ricci soliton with potential $\xi$ and constants $(\lambda, 0,-2 n-\lambda)$ if and only if the manifold is Einstein-like with constants $(-\lambda, 1, \lambda+2 n-1)$.

(ii) The manifold admits a shrinking Ricci soliton with potential $\xi$ and constant $-2 n$ if and only if the manifold is Einstein-like with constants $(2 n, 1,-1)$. 
(iii) The manifold is $\eta$-Einstein with constants $(a, 0,2 n-a)$ if and only if it admits a Ricci-like soliton with potential $\xi$ and constants $(-a, 1, a-2 n-1)$.

(iv) The manifold is Einstein with constant $2 n$ if and only if it admits a Riccilike soliton with potential $\xi$ and constants $(-2 n, 1,-1)$.

If $(M, \varphi, \xi, \eta, g)$ is Sasaki-like, we have

$$
\left(\mathcal{L}_{\xi} g\right)(x, y)=g\left(\nabla_{x} \xi, y\right)+g\left(x, \nabla_{y} \xi\right)=-2 g(x, \varphi y),
$$

i.e. $\frac{1}{2} \mathcal{L}_{\xi} g=-\tilde{g}+\eta \otimes \eta$. Then, because of (2.1), $\rho$ takes the form

$$
\rho=-\lambda g+(1-\mu) \tilde{g}-(1+\nu) \eta \otimes \eta .
$$

By direct computations from (2.3) we infer the following

Corollary 2.2. Let $(M, \varphi, \xi, \eta, g)$ satisfy the conditions in the general case of Theorem 2.1. Then, the constants $a, b, c, \lambda, \mu, \nu$ are expressed by scalar curvatures $\tau$ and $\tilde{\tau}$ with respect to $g$ and $\tilde{g}$, respectively, as follows

$$
\begin{array}{lll}
\lambda=1-\frac{1}{2 n} \tau, & \mu=2-\frac{1}{2 n} \tilde{\tau}, & \nu=\frac{1}{2 n}(\tau+\tilde{\tau})-2 n-3, \\
a=\frac{1}{2 n} \tau-1, & b=\frac{1}{2 n} \tilde{\tau}-1, & c=2 n+2-\frac{1}{2 n}(\tau+\tilde{\tau}) .
\end{array}
$$

Using (1.3), (1.4), (1.5), (1.6) and (2.2), we apply covariant derivatives to (2.3) and we get

$$
\begin{aligned}
\left(\nabla_{x} \rho\right)(y, z) & =(1-\mu)\{g(\varphi x, \varphi y) \eta(z)+g(\varphi x, \varphi z) \eta(y)\} \\
& +(\mu+\nu)\{g(x, \varphi y) \eta(z)+g(x, \varphi z) \eta(y)\} .
\end{aligned}
$$

Obviously, the tensors $\left(\nabla_{x} \rho\right)(\varphi y, \varphi z),\left(\nabla_{\xi} \rho\right)(y, z)$ and $\left(\nabla_{x} \rho\right)(\xi, \xi)$ vanish and therefore we establish the truthfulness of the following

Proposition 2.3. Every Einstein-like Sasaki-like manifold $(M, \varphi, \xi, \eta, g)$ admitting a Ricci-like soliton with potential $\xi$ is Ricci $\eta$-parallel and Ricci parallel along $\xi$, i.e. $\left.(\nabla \rho)\right|_{\operatorname{ker} \eta}=0$ and $\nabla_{\xi} \rho=0$, respectively.

Proposition 2.4. Let $(M, \varphi, \xi, \eta, g)$ be a Sasaki-like manifold admitting a Riccilike soliton with potential $\xi$ and constants $(\lambda, \mu, \nu)$. The manifold is locally Ricci symmetric if and only if $(\lambda, \mu, \nu)=(-2 n, 1,-1)$, i.e. it is an Einstein manifold.

Proof. The manifold is locally Ricci symmetric, i.e. $\left(\nabla_{x} \rho\right)(y, z)=0$, if and only if $1-\mu=\mu+\nu=0$, which is equivalent to $\mu=-\nu=1$. The value of $\lambda$ comes from the condition $\lambda+\mu+\nu=-2 n$ since the manifold is Sasaki-like. The conclusion that the manifold is Einstein follows from Theorem 2.1 (iv).

Let us remark that the Sasaki-like manifold is locally Ricci symmetric just in the case (iv) of Theorem 2.1.

Now for the Ricci tensor, we consider the term $\nabla$-recurrent, which is weaker than the usual parallelism. We say that the Ricci tensor is $\nabla$-recurrent if its covariant derivative with respect to $\nabla$, i.e. $\nabla \rho$, is expressed only by $\rho$ and some 1 -form.

Proposition 2.5. Let $(M, \varphi, \xi, \eta, g)$ be a Sasaki-like manifold admitting a Riccilike soliton with potential $\xi$ and constants $(\lambda, \mu, \nu)$ with the condition $(\lambda, \mu) \neq(0,1)$. Then the Ricci tensor $\rho$ of this manifold is $\nabla$-recurrent and satisfies the formula

$$
\begin{aligned}
\left(\nabla_{x} \rho\right)(y, z)= & \frac{(1-\mu)^{2}+\lambda(\lambda+2 n)}{\lambda^{2}+(1-\mu)^{2}}\{\rho(x, \varphi y) \eta(z)+\rho(x, \varphi z) \eta(y)\} \\
& -\frac{2(\lambda+n)(1-\mu)}{\lambda^{2}+(1-\mu)^{2}}\{\rho(\varphi x, \varphi y) \eta(z)+\rho(\varphi x, \varphi z) \eta(y)\} .
\end{aligned}
$$


Proof. The equality (2.3), by virtue of (1.1), (1.2), (1.3) and $\lambda+\mu+\nu=-2 n$, can be rewritten as

$$
\rho(x, y)=\lambda g(\varphi x, \varphi y)+(1-\mu) g(x, \varphi y)+2 n \eta(x) \eta(y)
$$

and therefore there are valid the following two equalities

$$
\begin{aligned}
& \rho(x, \varphi y)=-\lambda g(x, \varphi y)+(1-\mu) g(\varphi x, \varphi y), \\
& \rho(\varphi x, \varphi y)=-\lambda g(\varphi x, \varphi y)-(1-\mu) g(x, \varphi y) .
\end{aligned}
$$

The system of the latter two equations for $(\lambda, \mu) \neq(0,1)$ is solved with respect to $g(\varphi x, \varphi y)$ and $g(x, \varphi y)$ as follows

$$
\begin{aligned}
& g(x, \varphi y)=\frac{1}{\lambda^{2}+(1-\mu)^{2}}\{-\lambda \rho(x, \varphi y)+(1-\mu) \rho(\varphi x, \varphi y)\}, \\
& g(\varphi x, \varphi y)=\frac{1}{\lambda^{2}+(1-\mu)^{2}}\{-\lambda \rho(\varphi x, \varphi y)+(1-\mu) \rho(x, \varphi y)\} .
\end{aligned}
$$

Substituting the latter equalities to (2.4), we get the recurrent dependence (2.5) of the Ricci tensor.

\section{Ricci-like solitons With Potential Pointwise COlLinear With the REEB VECTOR FIELD ON SASAKI-LIKE MANIFOLDS}

Similarly to the definition of a Ricci-like soliton with potential $\xi$ by $(2.1)$, we introduce the following notion. We say that $(M, \varphi, \xi, \eta, g)$ admits a Ricci-like soliton with potential vector field $v$ if the following condition is satisfied for a triplet of constants $(\lambda, \mu, \nu)$

$$
\frac{1}{2} \mathcal{L}_{v} g+\rho+\lambda g+\mu \tilde{g}+\nu \eta \otimes \eta=0 .
$$

Suppose that $(M, \varphi, \xi, \eta, g)$ is a Sasaki-like manifold admitting a Ricci-like soliton whose potential vector field $v$ is pointwise collinear with $\xi$, i.e. $v=k \xi$, where $k$ is a differentiable function on $M$. It is clear that $k=\eta(v)$ and therefore $v$ belongs to the vertical distribution $H^{\perp}=\operatorname{span} \xi$, which is orthogonal to the contact distribution $H=\operatorname{ker} \eta$ with respect to $g$.

Theorem 3.1. Let $(M, \varphi, \xi, \eta, g), \operatorname{dim} M=2 n+1$, be a Sasaki-like manifold admitting a Ricci-like soliton with constants $(\lambda, \mu, \nu)$ whose potential vector field $v$ is pointwise collinear with the Reeb vector field $\xi$, i.e. $v=k \xi$, where $k$ is a differentiable function on $M$. Then we have $k=\mu$, i.e. $k$ is constant, the equality $\lambda+\nu=-k-2 n$ is valid and the manifold is $\eta$-Einstein with constants

$$
(a, b, c)=(-\lambda, 0, \lambda+2 n) .
$$

Proof. Due to the first equality in (1.7), the expression of $\mathcal{L}_{v} g$ in the considered case has the form

$$
\begin{aligned}
\left(\mathcal{L}_{v} g\right)(x, y) & =g\left(\nabla_{x} v, y\right)+g\left(x, \nabla_{y} v\right)=g\left(\nabla_{x} k \xi, y\right)+g\left(x, \nabla_{y} k \xi\right) \\
& =\mathrm{d} k(x) \eta(y)+\mathrm{d} k(y) \eta(x)-2 k g(x, \varphi y) .
\end{aligned}
$$

Replacing it in (3.1), we have

$$
\begin{aligned}
\mathrm{d} k(x) \eta(y)+\mathrm{d} k(y) \eta(x)=-2\{\rho(x, y) & +\lambda g(x, y)-(k-\mu) g(x, \varphi y) \\
& +(\mu+\nu) \eta(x) \eta(y)\} .
\end{aligned}
$$


Substituting $y$ for $\xi$ and using the expression of $\rho(x, \xi)$ from (1.7), the latter equality implies

$$
\mathrm{d} k(x)=-\{\mathrm{d} k(\xi)+2(\lambda+\mu+\nu+2 n)\} \eta(x),
$$

that yields the following equality for $x=\xi$

$$
\mathrm{d} k(\xi)=-(\lambda+\mu+\nu+2 n) .
$$

Therefore, (3.3) takes the form

$$
\mathrm{d} k(x)=-(\lambda+\mu+\nu+2 n) \eta(x) .
$$

Bearing in mind (3.4) and (3.2), we obtain the following expression of the Ricci tensor

$$
\rho=-\lambda g+(k-\mu) \tilde{g}+(\lambda+\mu+2 n-k) \eta \otimes \eta .
$$

The latter equality is the condition for manifold to be almost Einstein-like with functions

$$
(a, b, c)=(-\lambda, k-\mu, \lambda+\mu+2 n-k) .
$$

Then, according to Proposition 1.1, $(M, \varphi, \xi, \eta, g)$ is $\eta$-Einstein with constants

$$
(a, b, c)=\left(\frac{\tau}{2 n}-1,0,2 n+1-\frac{\tau}{2 n}\right) .
$$

Comparing the two triads $(a, b, c)$ from the above, we infer that $k=\mu$, i.e. $k$ is a constant.

Thus, from the formula in (3.4) we deduce that the condition

$$
\lambda+\mu+\nu=-2 n
$$

is satisfied. Then, equality (3.5) becomes

$$
\rho=-\lambda g+(\lambda+2 n) \eta \otimes \eta,
$$

which completes the proof.

3.1. Additional curvature properties. Let $(M, \varphi, \xi, \eta, g), \operatorname{dim} M=2 n+1$, be a Sasaki-like manifold admitting a Ricci-like soliton with vertical potential $v$, i.e. $v=k \xi, k=$ const. Then, according to Theorem 3.1, the soliton constants are $(\lambda, k,-\lambda-k-2 n)$ and $(M, \varphi, \xi, \eta, g)$ is $\eta$-Einstein with constants $(a, b, c)=$ $(-\lambda, 0, \lambda+2 n)$, where $\lambda=1-\frac{1}{2 n} \tau$.

If consider the condition for locally Ricci symmetry, i.e. $\nabla \rho$ vanishes, we immediately obtain the following

Proposition 3.2. Let $(M, \varphi, \xi, \eta, g)$ be a Sasaki-like manifold admitting a Riccilike soliton with vertical potential. The manifold is locally Ricci symmetric if and only if it is an Einstein manifold.

Proof. By virtue of (3.6), in similar way as for (2.4), we obtain in this case

$$
\left(\nabla_{x} \rho\right)(y, z)=-(\lambda+2 n)\{g(x, \varphi y) \eta(z)+g(x, \varphi z) \eta(y)\} .
$$

Then, the statement is easy to conclude.

Let us impose the condition $R(\xi,.) \cdot \rho=0$, i.e. it is valid the following

$$
\rho(R(\xi, x) y, z)+\rho(y, R(\xi, x) z)=0 .
$$


Proposition 3.3. Let $(M, \varphi, \xi, \eta, g)$ be a Sasaki-like manifold admitting a Riccilike soliton with vertical potential. The condition $R(\xi,.) \cdot \rho=0$ is satisfied if and only if the manifold is Einstein.

Proof. Then, because of (1.7), equality (3.8) follows

$$
\rho(x, y) \eta(z)+\rho(x, z) \eta(y)-2 n g(x, y) \eta(z)-2 n g(x, z) \eta(y)=0 .
$$

By virtue of (3.6) and (3.9), we obtain

$$
(\lambda+2 n)\{g(\varphi x, \varphi y) \eta(z)+g(\varphi x, \varphi z) \eta(y)\}=0 .
$$

Equality (3.10) for $z=\xi$ provides $(\lambda+2 n) g(\varphi x, \varphi y)=0$, which is equivalent to

$$
\lambda=-2 n .
$$

Therefore, condition (3.8) implies that the manifold is Einstein.

A non-vanishing Ricci tensor $\rho$ is called cyclic parallel or of Codazzi type if it satisfies the condition [10]

$$
\left(\nabla_{x} \rho\right)(y, z)+\left(\nabla_{y} \rho\right)(z, x)+\left(\nabla_{z} \rho\right)(x, y)=0
$$

or

respectively.

$$
\left(\nabla_{x} \rho\right)(y, z)=\left(\nabla_{y} \rho\right)(x, z),
$$

It is said that a non-vanishing Ricci operator $Q$ is Ricci $\varphi$-symmetric if it satisfies the condition [6]

$$
\varphi^{2}\left(\nabla_{x} Q\right) y=0 .
$$

In [9], if the latter condition is satisfied for arbitrary vector fields on the manifold, it is called globally Ricci $\varphi$-symmetric, and when these vector fields are orthogonal to $\xi$, then the manifold is called locally Ricci $\varphi$-symmetric.

Similarly to Proposition 3.3, we establish the truthfulness of the following

Proposition 3.4. Let $(M, \varphi, \xi, \eta, g)$ be a Sasaki-like manifold admitting a Ricci-like soliton with vertical potential.

(i) The manifold has a cyclic parallel Ricci tensor if and only if it is Einstein.

(ii) The Ricci tensor is of Codazzi type if and only the manifold is Einstein.

(iii) The manifold is locally Ricci $\varphi$-symmetric.

(iv) The manifold is globally Ricci $\varphi$-symmetric if and only if it is Einstein.

In [5], the notion of almost pseudo Ricci symmetric manifolds is introduced by the following condition for its non-vanishing Ricci tensor

$$
\left(\nabla_{x} \rho\right)(y, z)=\{\alpha(x)+\beta(x)\} \rho(y, z)+\alpha(y) \rho(x, z)+\alpha(z) \rho(x, z),
$$

where $\alpha$ and $\beta$ are non-vanishing 1-forms.

Proposition 3.5. Let $(M, \varphi, \xi, \eta, g)$ be a Sasaki-like manifold admitting a Ricci-like soliton with vertical potential. Then the manifold is almost pseudo Ricci symmetric if and only if the manifold is Einstein.

Proof. Substituting (3.7) in the defining condition (3.11) for an almost pseudo Ricci symmetric manifold gives us the following equality

$$
\begin{aligned}
& \{\alpha(x)+\beta(x)\}\{\lambda g(\varphi y, \varphi z)+2 n \eta(y) \eta(z)\} \\
& +\alpha(y)\{\lambda g(\varphi x, \varphi z)+2 n \eta(x) \eta(z)\}+\alpha(z)\{\lambda g(\varphi x, \varphi y)+2 n \eta(x) \eta(y)\} \\
& +(\lambda+2 n)\{g(x, \varphi y) \eta(z)+g(x, \varphi z) \eta(y)\}=0
\end{aligned}
$$


Setting successively $x, y$ and $z$ as $\xi$ in the latter equality and then combining the obtained equalities, we get

$$
\alpha=\alpha(\xi) \eta, \quad \beta=-2 \alpha(\xi) \eta .
$$

Imposing these equalities in (3.12) gives

$$
\begin{aligned}
& \lambda \alpha(\xi)\{-2 g(\varphi y, \varphi z) \eta(x)+g(\varphi x, \varphi z) \eta(y)+g(\varphi x, \varphi y) \eta(z)\} \\
& +(\lambda+2 n)\{g(x, \varphi y) \eta(z)+g(x, \varphi z) \eta(y)\}=0,
\end{aligned}
$$

which for $z=\xi$ implies

$$
\lambda \alpha(\xi) g(\varphi x, \varphi y)+(\lambda+2 n) g(x, \varphi y)=0 .
$$

The latter equality is fulfilled if and only if $\lambda=-2 n$ and $\alpha(\xi)=0$.

Vice versa, let $(M, \varphi, \xi, \eta, g)$ be Einstein, i.e. $\rho=2 n g$. Then, equality (3.11) takes the following form

$$
\{\alpha(x)+\beta(x)\} g(y, z)+\alpha(y) g(x, z)+\alpha(z) g(x, y)=0 .
$$

Again by setting successively $x, y$ and $z$ as $\xi$ and combining the obtained equalities, we get the formulas (3.13) and then (3.14) implies

$$
\alpha(\xi)\{-2 \eta(x) g(y, z)+\eta(y) g(x, z)+\eta(z) g(x, y)\}=0
$$

for arbitrary $x, y, z$ and therefore $\alpha(\xi)=0$ holds. Thus, (3.11) is satisfied providing $\alpha(\xi)$ vanishes, which completes the proof.

A manifold is called special weakly Ricci symmetric if its non-vanishing Ricci tensor satisfies the following condition [21]

$$
\left(\nabla_{x} \rho\right)(y, z)=2 \alpha(x) \rho(y, z)+\alpha(y) \rho(x, z)+\alpha(z) \rho(x, z),
$$

where $\alpha$ is a non-vanishing 1 -form.

Proposition 3.6. Let $(M, \varphi, \xi, \eta, g)$ be a Sasaki-like manifold admitting a Ricci-like soliton with vertical potential. Then the manifold is special weakly Ricci symmetric if and only if the manifold is Einstein.

Proof. Comparing (3.15) with (3.11), we deduce that an almost pseudo Ricci symmetric manifold with $\alpha=\beta$ is a special weakly Ricci symmetric manifold. Then, (3.13) implies $\alpha=0$ and therefore the manifold has $\nabla \rho=0$. By virtue of Proposition 3.5 , we conclude the validity of the statement.

Finally in the series, for the studied manifold $(M, \varphi, \xi, \eta, g)$, let us consider the curvature condition $Q \cdot R=0$, which can read as

$$
R(x, y, z, Q w)-R(Q x, y, z, w)-R(x, Q y, z, w)-R(x, y, Q z, w)=0 .
$$

Proposition 3.7. Let $(M, \varphi, \xi, \eta, g)$ be a Sasaki-like manifold with the curvature property $Q \cdot R=0$. Then the manifold does not admit a Ricci-like soliton with vertical potential.

Proof. Taking the trace of (3.16) for $x=e_{i}, w=e_{j}$ and due to the symmetry of $Q$, we obtain

$$
\rho(Q y, z)+\rho(y, Q z)=0 .
$$

After that we use (3.6) and get

$$
\lambda^{2} g(\varphi y, \varphi z)-8 n^{2} \eta(y) \eta(z)=0,
$$

which has no solution. 


\section{Parallel Symmetric SECOND-ORDER COVARIANT TENSOR ON A SASAKI-LIKE ALMOST CONTACT B-METRIC MANIFOLD}

In [7], L.P. Eisenhart proved that if a positive definite Riemannian manifold admits a second-order parallel symmetric tensor other than a constant multiple of the metric tensor, then it is reducible. In [14], H. Levy proved that a second-order parallel symmetric non-singular (with non-vanishing determinant) tensor in a space of constant curvature is proportional to the metric tensor. In [22], R. Sharma proved a generalization over Levy's theorem for dimension greater than two in non-flat real space forms.

Sasaki-like almost contact B-metric manifolds are not need to be real space forms in general. We now prove an assertion of the kind of the theorems above.

Proposition 4.1. On an arbitrary Sasaki-like manifold, every symmetric secondorder covariant tensor that is parallel with respect to the Levi-Civita connection of the B-metric, is a constant multiple of this metric.

Proof. Let $h$ be a symmetric $(0,2)$-tensor field which is parallel, i.e. $\nabla h=0$ with respect to the Levi-Civita connection of $g$. We use the Ricci identity for $h$, i.e.

$$
\left(\nabla_{x} \nabla_{y} h\right)(z, w)-\left(\nabla_{y} \nabla_{x} h\right)(z, w)=-h(R(x, y) z, w)-h(z, R(x, y) w) .
$$

Since $\nabla h=0$, the latter identity implies

$$
h(R(x, y) z, w)+h(z, R(x, y) w)=0
$$

and therefore the following property is valid

$$
h(R(x, y) \xi, \xi)=0 .
$$

Let the considered manifold be Sasaki-like. Then, we replace the expression of $R(x, y) \xi$ from $(1.7)$ in $(4.1)$ and we obtain

$$
h(x, \xi) \eta(y)-h(y, \xi) \eta(x)=0 .
$$

If we put $y=\xi$ in the latter equality, it follows

$$
h(x, \xi)=h(\xi, \xi) \eta(x) .
$$

Since $\eta\left(\nabla_{x} \xi\right)$ vanishes because of $g(\xi, \xi)=1$, then (4.2) implies

$$
h\left(\nabla_{x} \xi, \xi\right)=0 .
$$

From the expression of $\left(\nabla_{x} h\right)(y, z)$ in the case $\nabla h=0$ and $y=z=\xi$ we have

$$
x(h(\xi, \xi))=2 h\left(\nabla_{x} \xi, \xi\right),
$$

which means that $h(\xi, \xi)$ is a constant because of (4.3).

Next, we take the covariant derivative of (4.2) with respect to $y$ and we get $h(x, \varphi y)=h(\xi, \xi) g(x, \varphi y)$, bearing in mind the first equality of (1.7). We substitute $y$ for $\varphi y$ in the latter equality for $h$ and use (1.1) and (4.2) to obtain the following

$$
h(x, y)=h(\xi, \xi) g(x, y) .
$$

The latter equality means that $h$ is a constant multiple of $g$ and therefore we proved the following

Let us apply this assertion to a Ricci-like soliton. 
Theorem 4.2. Let $(M, \varphi, \xi, \eta, g)$ be a Sasaki-like manifold of dimension $2 n+1$ and let $h$ be the tensor $\frac{1}{2} \mathcal{L}_{\xi} g+\rho+\mu \tilde{g}+\nu \eta \otimes \eta$, where $\mu, \nu \in \mathbb{R}$. The tensor $h$ is parallel with respect to $\nabla$ of $g$ if and only if $(M, \varphi, \xi, \eta, g)$ admits a Ricci-like soliton with potential $\xi$ and constants $(\lambda, \mu, \nu)$, where $\lambda=-h(\xi, \xi)=-\mu-\nu-2 n$.

Proof. Since $h=\frac{1}{2} \mathcal{L}_{\xi} g+\rho+\mu \tilde{g}+\nu \eta \otimes \eta$ and for Sasaki-like manifolds we have $\left(\mathcal{L}_{\xi} g\right)(x, y)=g\left(\nabla_{x} \xi, y\right)+g\left(x, \nabla_{y} \xi\right)=-2 g(x, \varphi y)$, i.e. $\frac{1}{2} \mathcal{L}_{\xi} g=-\tilde{g}+\eta \otimes \eta$. Then $h$ takes the form

$$
h=\rho+(\mu-1) \tilde{g}+(\nu+1) \eta \otimes \eta .
$$

Now, let $h$ be parallel. According to Proposition $4.1, h=h(\xi, \xi) g$ holds true in this case. From (4.4) and the last equality in (1.7), we obtain $h(\xi, \xi)=2 n+\mu+\nu$ and therefore $h=(2 n+\mu+\nu) g$. The latter equality and (2.1) imply that there is a Ricci-like soliton with constants $(\lambda, \mu, \nu)$, where $\lambda=-\mu-\nu-2 n$.

Vice versa, let $(M, \varphi, \xi, \eta, g)$ admit a Ricci-like soliton with constants $(\lambda, \mu, \nu)$, i.e. (2.1) is valid. The latter condition can be rewritten as $h=-\lambda g$. Bearing in mind that $\lambda$ is constant and $g$ is parallel, it follows that $h$ is parallel with respect to $\nabla$, too.

\section{EXAmple of an Einstein-like Sasaki-Like manifold, ADmitTing A RICCI-LIKE SOLITON WITH POTENTIAL REEB VECTOR FIELD}

In Example 2 of [13], it is given a Lie group $G$ of dimension 5 (i.e. $n=2$ ) with a basis of left-invariant vector fields $\left\{e_{0}, \ldots, e_{4}\right\}$ and the corresponding Lie algebra is defined by the commutators

$$
\begin{array}{lll}
{\left[e_{0}, e_{1}\right]=p e_{2}+e_{3}+q e_{4},} & {\left[e_{0}, e_{2}\right]=-p e_{1}-q e_{3}+e_{4},} & \\
{\left[e_{0}, e_{3}\right]=-e_{1}-q e_{2}+p e_{4},} & {\left[e_{0}, e_{4}\right]=q e_{1}-e_{2}-p e_{3}, \quad p, q \in \mathbb{R} .}
\end{array}
$$

Then, $G$ is equipped with an almost contact B-metric structure by

$$
\begin{aligned}
& g\left(e_{0}, e_{0}\right)=g\left(e_{1}, e_{1}\right)=g\left(e_{2}, e_{2}\right)=-g\left(e_{3}, e_{3}\right)=-g\left(e_{4}, e_{4}\right)=1, \\
& g\left(e_{i}, e_{j}\right)=0, \quad i, j \in\{0,1, \ldots, 4\}, i \neq j, \\
& \xi=e_{0}, \quad \varphi e_{1}=e_{3}, \quad \varphi e_{2}=e_{4}, \quad \varphi e_{3}=-e_{1}, \quad \varphi e_{4}=-e_{2} .
\end{aligned}
$$

There are computed the components of $\nabla$ for $g$ and the non-zero of them are the following

$$
\begin{array}{cl}
\nabla_{e_{0}} e_{1}=p e_{2}+q e_{4}, & \nabla_{e_{0}} e_{2}=-p e_{1}-q e_{3}, \\
\nabla_{e_{0}} e_{3}=-q e_{2}+p e_{4}, & \nabla_{e_{0}} e_{4}=q e_{1}-p e_{3}, \\
\nabla_{e_{1}} e_{0}=-e_{3}, \quad \nabla_{e_{2}} e_{0}=-e_{4}, & \nabla_{e_{3}} e_{0}=e_{1}, \quad \nabla_{e_{4}} e_{0}=e_{2}, \\
\nabla_{e_{1}} e_{3}=\nabla_{e_{2}} e_{4}=\nabla_{e_{3}} e_{1}=\nabla_{e_{4}} e_{2}=-e_{0} .
\end{array}
$$

It is verified that the constructed almost contact B-metric manifold $(G, \varphi, \xi, \eta, g)$ is Sasaki-like.

The components of the curvature tensor $R_{i j k l}=g\left(R\left(e_{i}, e_{j}\right) e_{k}, e_{l}\right)$ and those of the Ricci tensor $\rho_{i j}=\rho\left(e_{i}, e_{j}\right)$ are computed for the same manifold in [16]. The non-zero of them are determined by the following equalities and the usual properties $R_{i j k l}=-R_{j i k l}=-R_{i j l k}$ :

$$
\begin{aligned}
& R_{0110}=R_{0220}=R_{1234}=R_{1432}=R_{2341}=R_{3412}=R_{1331}=R_{2442}=1, \\
& R_{0330}=R_{0440}=-1, \quad \rho_{00}=4 .
\end{aligned}
$$


Since $\rho=4 \eta \otimes \eta$ is satisfied, then $(G, \varphi, \xi, \eta, g)$ is $\eta$-Einstein with constants

$$
(a, b, c)=(0,0,4) \text {. }
$$

Moreover, it is clear that $\tau=\tilde{\tau}=4$.

Also, there are computed the components $\left(\mathcal{L}_{\xi} g\right)_{i j}=\left(\mathcal{L}_{\xi} g\right)\left(e_{i}, e_{j}\right)$ and the nonzero of them are the following $\left(\mathcal{L}_{\xi} g\right)_{13}=\left(\mathcal{L}_{\xi} g\right)_{24}=\left(\mathcal{L}_{\xi} g\right)_{31}=\left(\mathcal{L}_{\xi} g\right)_{42}=2$, which means that $\mathcal{L}_{\xi} g=-2 \tilde{g}+2 \eta \otimes \eta$.

After that, it is obtained that (3.1) is satisfied for

$$
(\lambda, \mu, \nu)=(0,1,-5)
$$

and therefore $(G, \varphi, \xi, \eta, g)$ admits a Ricci-like soliton with potential $\xi$. Therefore, the condition $\lambda+\mu+\nu=-2 n$ is fulfilled and this is in unison with Theorem 2.1.

Now, using (5.1) and the only non-zero component $\rho_{00}=4$ of $\rho$, we compute the components of $\left(\nabla_{i} \rho\right)_{j k}=\left(\nabla_{e_{i}} \rho\right)\left(e_{j}, e_{k}\right)$ of $\nabla \rho$ and the non-zero ones of them are the following

$$
\left(\nabla_{1} \rho\right)_{30}=\left(\nabla_{2} \rho\right)_{40}=\left(\nabla_{3} \rho\right)_{10}=\left(\nabla_{4} \rho\right)_{20}=4
$$

and their symmetric about $j$ and $k$. These results are in accordance with Proposition 2.3 .

In conclusion, the constructed 5-dimensional example of a Sasaki-like manifold with the results in (5.2) and (5.3) supports also Theorem 2.1, Theorem 3.1 for $k=1$ and it agrees with Proposition 4.1 and Theorem 4.2 for the trivial case $h=0$.

Acknowledgment. The author was supported by projects MU19-FMI-020 and FP19-FMI-002 of the Scientific Research Fund, University of Plovdiv Paisii Hilendarski, Bulgaria.

\section{REFERENCES}

[1] C.S. Bagewadi, G. Ingalahalli, Ricci solitons in Lorentzian $\alpha$-Sasakian manifolds, Acta Math. Acad. Paedagog. Nyházi. (N.S.) 28 (2012) 59-68. 1

[2] C.S. Bagewadi, G. Ingalahalli, Certain results on Ricci solitons in trans-Sasakian manifolds, J. Math. 2013 art. ID 787408 (10 pages). 1

[3] A. M. Blaga, $\eta$-Ricci solitons on Lorentzian para-Sasakian manifolds, Filomat 30 (2) (2016) 489-496. 1

[4] A. M. Blaga, S. Y. Perktaş, B. E. Acet, F. E. Erdoğan, $\eta$-Ricci solitons in $(\varepsilon)$-almost paracontact metric manifolds, Glas. Mat. Ser. III 53 (73) (2018) 205-220. 1

[5] M. C. Chaki, T. Kawaguchi, On almost pseudo Ricci symmetric manifolds, Tensor N.S. 68 (2007) 10-14. 8

[6] U. C. De, A. Sarkar, On $\varphi$-Ricci symmetric Sasakian manifolds, Proc. Jangjeon Math. Soc. 11 (2008) 47-52. 8

[7] L.P. Eisenhart, Symmetric tensors of the second order whose first covariant derivatives are zero, Trans. Amer. Math. Soc. 25 (1923) 297-306. 10

[8] G. Ganchev, V. Mihova, K. Gribachev, Almost contact manifolds with B-metric, Math. Balkanica (N.S.) 7 (1993) 261-276. 1, 2

[9] S. Ghosh, U. C. De, On $\phi$-Ricci symmetric $(\kappa, \mu)$-contact metric manifolds, Acta Math. Univ. Comenianae 86 (2) (2017) 205-213. 8

[10] A. Gray, Einstein-like manifolds which are not Einstein, Geom. Dedicata 7 (1978) 259-280. 8

[11] R.S. Hamilton, Three-manifolds with positive Ricci curvature, J. Differential Geom. 17 (1982) 255-306. 1

[12] G. Ingalahalli, C.S. Bagewadi, Ricci solitons in $\alpha$-Sasakian manifolds, Int. Sch. Res. Notices Geometry 2012 art. ID 421384 (2012) (13 pages). 1

[13] S. Ivanov, H. Manev, M. Manev, Sasaki-like almost contact complex Riemannian manifolds, J. Geom. Phys. 105 (2016) 136-148. 3, 11 
[14] H. Levy, Symmetric tensors of the second order whose covariant derivatives vanish, Ann. of Math. (2) 27 (1926) 91-98. 10

[15] M. Manev, Properties of curvature tensors on almost contact manifolds with B-metric. Sci. Works V. Levski Higher Mil. School, Veliko Tarnovo 27 (1993) 221-227. 3

[16] M. Manev, Ricci-like solitons on almost contact B-metric manifolds, arXiv:1912.09921. 1, 3, 4,11

[17] M. Manev, Ricci-like solitons with arbitrary potential and gradient almost Ricci-like solitons on Sasaki-like almost contact B-metric manifolds, arXiv:2003. 4

[18] M. Manev, K. Gribachev, Contactly conformal transformations of almost contact manifolds with B-metric Serdica Math. J. 19 (1993) 287-299. 1

[19] K. Matsumoto, On Lorentzian paracontact manifolds, Bull. Yamagata Univ. Natur. Sci. 12 (1989) 151-156. 1

[20] H. G. Nagaraja, C.R. Premalatha, Ricci solitons in Kenmotsu manifolds, J. Math. Anal. 3 (2) (2012) 18-24. 1

[21] H. Singh, Q. Khan, On special weakly symmetric Riemannian manifolds, Publ. Math. Debrecen 58 (3) (2001) 523-536. 9

[22] R. Sharma, Second order parallel tensor in real and complex space forms, Int. J. Math. Math. Sci. 12 (4) (1989) 787-790. 10

[23] R. Sharma, Certain results on K-contact and $(\kappa, \mu)$-contact manifolds, J. Geom. 89 (1-2) (2008) 138-147. 1

[24] S. K. Yadav, A. Kushwaha, D. Narain, Certain results for $\eta$-Ricci solitons and Yamabe solitons on quasi-Sasakian 3-manifolds, Cubo 21 (2) (2019) 77-98. 1

University of Plovdiv Paisi Hilendarski, Faculty of Mathematics and Informatics, Department of Algebra and Geometry, 24 Tzar Asen St., Plovdiv 4000, Bulgaria \& Medical University of Plovdiv, Faculty of Public Health, Department of Medical Informatics, Biostatistics and E-Learning, 15A Vasil Aprilov Blvd., Plovdiv 4002, BulGARIA

E-mail address: mmanev@uni-plovdiv.bg 